\title{
Lack of effects of tomato products on endothelial function in human subjects: results of a randomised, placebo-controlled cross-over study
}

\author{
Verena Stangl ${ }^{1 *}$, Claudia Kuhn ${ }^{1}$, Sonja Hentschel ${ }^{1}$, Nicoline Jochmann ${ }^{1}$, Christine Jacob ${ }^{1}$, Volker Böhm², \\ Kati Fröhlich ${ }^{2}$, Lars Müller ${ }^{2}$, Christine Gericke ${ }^{3}$ and Mario Lorenz ${ }^{1}$ \\ ${ }^{1}$ Medizinische Klinik für Kardiologie und Angiologie, Campus Mitte, Charité - Universitätsmedizin, Berlin, Germany \\ ${ }^{2}$ Institut für Ernährungswissenschaften, Friedrich-Schiller-Universität, Jena, Germany \\ ${ }^{3}$ Institut für Biometrie und Klinische Epidemiologie, Campus Mitte, Charité - Universitätsmedizin, Berlin, Germany
}

(Received 7 April 2010 - Revised 19 July 2010 - Accepted 29 July 2010 - First published online 24 August 2010)

\section{Abstract}

Epidemiological studies suggest that consumption of tomato products reduces the risk of CVD via antioxidant, hypocholesterolaemic and anti-inflammatory mechanisms. Although experimental data also describe beneficial effects on endothelial function, clinical data in human subjects are lacking. To test the hypothesis that tomato ingestion ameliorates endothelial function, we randomised healthy non-smoking postmenopausal women to consume a buttered roll with and without tomato purée $(70 \mathrm{~g})$ in a cross-over design. Endothelial-dependent flow-mediated dilation (FMD) and endothelial-independent nitro-mediated dilation of the brachial artery were assessed with highresolution ultrasound ( $13 \mathrm{MHz}$ linear array transducer). Acute $(24 \mathrm{~h})$ and long-term $(7 \mathrm{~d})$ effects were examined after daily consumption of the described meal. Nineteen volunteers completed the protocol and provided technically suitable ultrasound measurement data. Plasma lycopene levels increased from 0.30 (SEM 0.04) (baseline) to 0.42 (sem 0.04) and to 0.74 (SEM 0.06) $\mu \mathrm{M}$ after $24 \mathrm{~h}$ and $7 \mathrm{~d}$, respectively, with tomato purée consumption. These data indicated an effective absorption of the tomato product. However, both acute and long-term tomato purée consumption had no effects on endothelium-dependent or -independent dilation of the brachial artery. In addition, we found no correlation between lycopene plasma levels and FMD. In conclusion, consumption of tomato products associated with a significant increase in plasma lycopene levels had no effects on endothelial function in healthy postmenopausal women.

\section{Key words: Tomato products: Lycopene: Endothelial function: Postmenopausal women}

Several lines of evidence from epidemixological and intervention studies suggest that dietary intake of tomatoes and tomato products is associated with decreased risk of CVD $^{(1-4)}$. In a multicentre case-control study in ten European countries, plasma levels of lycopene, which represents more than $80 \%$ of total tomato carotenoids, were found to be protective with regard to acute myocardial infarction $^{(2)}$. In corroboration, low lycopene concentrations are evidently associated with an increased incidence of CVD.

Compelling experimental data support the protective role of tomato ingredients against processes involved in atherogenesis. Antioxidant effects of lycopene play a key role $^{(5,6)}$. Lycopene has free radical-scavenging properties and accordingly reduces oxidative stress ${ }^{(7)}$. Dietary supplementation of lycopene has been associated with reduction in lipid peroxidation and LDL oxidation ${ }^{(5,6,8,9)}$. In addition, a number of other anti-atherogenic mechanisms have been reported. Lycopene inhibited cytokine-induced expression of vascular cell adhesion molecule- 1 and intercellular cell adhesion molecule-1, thereby reducing monocyte adhesion to human endothelial cells ${ }^{(10,11)}$. Lycopene showed antiproliferative properties in smooth muscle cells by preventing platelet-derived growth factor-BB-induced migration and proliferation ${ }^{(12)}$. Furthermore, lycopene exerted a hypocholesterolaemic effect by inhibiting cholesterol synthesis $^{(13)}$.

There is some evidence that tomato carotenoids also have beneficial effects on endothelial function, which plays a key role in vascular homoeostasis. Serum lycopene concentrations were inversely related to soluble intercellular adhesion molecule-1, a marker of endothelial function and inflammation ${ }^{(14,15)}$. Lycopene supplementation prevented endothelial dysfunction in mice fed an atherogenic diet $^{(16)}$. Until now, it remains unclear whether tomato ingredients could have an impact on endothelial function

Abbreviations: FMD, flow-mediated dilation; MeOH, methanol; MTBE, methyl tert-butyl-diethyl ether. 
in humans. The aim of the present study was therefore to investigate whether acute or long-term consumption of tomato products ameliorates peripheral endothelial function in human volunteers in a randomised, crossover dietary intervention study.

\section{Subjects and methods}

\section{Study design}

Non-smoking healthy postmenopausal women were recruited by press advertisements. A total of thirty-one women were invited for clinical examination. Subjects with cardiovascular risk factors such as high blood cholesterol, diabetes, arterial hypertension and obesity were excluded. All measures of clinical parameters were required to lie within normal ranges for study inclusion: lipid profile, blood pressure, BMI, as well as routine internal and endocrine parameters. Eight subjects were excluded for not meeting the inclusion criteria. Three subjects refused to participate. Twenty subjects received the allocated interventions, one person did not yield evaluable endothelial function measurements. A total of nineteen healthy women completed the study. None of the participants had taken medication for at least 3 months before entering the study. The participants were asked not to consume tomato products at least 2 weeks before and during the study.

Study subjects consumed a buttered roll with or without tomato purée $(70 \mathrm{~g})$ in the morning after an overnight fast, in accordance with computer-generated randomisation numbers in a cross-over study design. Lycopene content was determined in the commercially available tomato purée used in the study (Smart Price Tomatenmark, Wal-Mart, Germany). Products from one batch were used throughout the study. Endothelial function was assessed at six time points for each patient: at baseline, $24 \mathrm{~h}$ and $7 \mathrm{~d}$ after consumption of a buttered roll with tomato purée (tomato period); second baseline after $14 \mathrm{~d}$ of tomato-free diet; $24 \mathrm{~h}$ and $7 \mathrm{~d}$ after daily consumption of a buttered roll without tomato purée (control period). At all six time points, blood pressure and heart rate were assessed at rest, and blood was collected for assessment of lycopene levels and measurement of plasma levels for total cholesterol, HDL-cholesterol, LDL-cholesterol and TAG. The study was approved by the Charité University Hospital Ethics Committee, and participants provided their written informed consent. The study was carried out in accordance with The Code of Ethics of the World Medical Association (Declaration of Helsinki).

\section{Determination of lycopene plasma levels}

Carotenoids were extracted according to Bieri et al. ${ }^{(17)}$, and with slight modification ${ }^{(18)}$. Echinenone (CaroteNature, Lupsingen, Switzerland) was used as internal standard.
All the experiments were carried out under subdued light to prevent photodegradation and isomerisation. The plasma extracts were analysed on a $\mathrm{C}_{30}(250 \times 4.6 \mathrm{~mm}$, $5 \mu \mathrm{m}$ ) column (Trentec, Rutesheim, Germany), preceded by a $\mathrm{C}_{18}$ ProntoSil $120-5-\mathrm{C} 18 \mathrm{H}(10 \times 4.0 \mathrm{~mm}, 5 \mu \mathrm{m})$ guard column (Bischoff, Leonberg, Germany) at $10 \pm 1^{\circ} \mathrm{C}$ with diode array detection at $470 \mathrm{~nm}$. The mobile phase $(1.0 \mathrm{ml} / \mathrm{min})$ consisted of methanol $(\mathrm{MeOH})$ and methyl tert-butyl-diethyl ether (MTBE). The following gradient procedure was used: (1) initial condition with $90 \%$ $\mathrm{MeOH}$ and $10 \% \mathrm{MTBE}$; (2) a $40 \mathrm{~min}$ linear gradient to $50 \% \mathrm{MeOH}$ and $50 \% \mathrm{MTBE}$; (3) $50 \% \mathrm{MeOH}$ and $50 \%$ MTBE for $20 \mathrm{~min}$; (4) a $10 \mathrm{~min}$ linear gradient to $90 \%$ $\mathrm{MeOH}$ and 10\% MTBE; (5) $90 \% \mathrm{MeOH}$ and 10\% MTBE for $10 \mathrm{~min}$. Lycopene was quantified using (all-E)-lycopene reference material (CaroteNature, Lupsingen, Switzerland). The concentration of the lycopene stock solution was checked periodically in $n$-hexane at $472 \mathrm{~nm}$ by using its extinction coefficient $E_{(1 \%, 1 \mathrm{~cm})}: 3450^{(19)}$. The plasma lycopene levels were given as total lycopene (sum of all detected lycopene isomers).

\section{Measurement of endothelial function of the brachial artery}

Endothelial function was measured by high-resolution vascular ultrasound with a $13 \mathrm{MHz}$ linear array transducer (Sonoline An-tares, Siemens, Germany) as recently described $^{(20)}$. Briefly, endothelium-dependent flow-mediated dilation (FMD) was assessed by measuring the change in brachial artery diameter for 2 min after reactive hyperaemia for $5 \mathrm{~min}$, according to the established guidelines ${ }^{(21,22)}$. Endothelium-independent nitro-mediated dilation was measured after sublingual application of nitroglycerine spray $(0.4 \mathrm{mg})$ for $6 \mathrm{~min}$. To improve the accuracy and reproducibility of the measurements, an attached device fixed the ultrasound probe at one site of the brachial artery during the entire period of measurement. FMD and nitro-mediated dilation were defined as the maximum percentage change in brachial artery diameter compared with baseline measurement. Analyses of diameter changes were conducted offline (Tom Tec Imaging Systems, Unterschleissheim, Germany) by two different investigators blinded to subject treatment.

\section{Statistical analysis}

All the statistical analyses were two-sided with the level of significance accepted at $P<0.05$. A linear mixed model with random effects for the control period and without random effects for the lycopene period (all participants had a positive slope over the three time points) was used for comparisons of plasma lycopene contents between the time points (time as fixed effect). Spearman's correlations were used for determinations of intra- and interobserver variability in endothelial function measurements and 
for associations between FMD and plasma lycopene levels. Statistical analysis was performed using SPSS, release 15.0 (SPSS, Inc., Chicago, IL, USA). The linear mixed models were programmed with $\mathrm{R}$ (version 2.6.2).

\section{Results}

A total of nineteen postmenopausal healthy women (mean age 58.9 (SD 6.3) years) finished the study protocol. Baseline characteristics of the study group are depicted in Table 1. Lycopene content of the tomato purée was determined before the start of the study: $70 \mathrm{~g}$ of the purée contained $46.2 \mathrm{mg}$ of lycopene. Thus, the volunteers consumed $46.2 \mathrm{mg}$ of lycopene/d. Plasma levels of lycopene at baseline and after consumption of $70 \mathrm{~g}$ tomato purée/d are shown in Fig. 1. A slight decrease in lycopene plasma levels was observed after $7 \mathrm{~d}$ in the control group. However, compared with controls, consumption of tomato purée resulted in an increase of lycopene plasma levels by $38 \%$ after $24 \mathrm{~h}$ and by $145 \%$ after $7 \mathrm{~d}$.

Owing to the cross-over design of the study, endothelial function was assessed at each time point with tomato purée consumption (tomato period) and without tomato purée (control period) for all the subjects. The intra- and interobserver variabilities were 0.99 and 0.98 for baseline diameter of the arteria brachialis and 0.99 and 0.99 for maximum dilation (FMD), respectively. FMD levels at baselines (control and tomato periods) revealed a broad inter-individual variation, ranging from 2.9 to $14.8 \%$. Mean baseline FMD was $7 \cdot 8$ (SEM 3.3 )\% before the tomato period and 7.7 (SEM 3.2)\% before the control period. Compared with baseline, there were no significant differences in FMD after acute $(24 \mathrm{~h})$ or chronic $(7 \mathrm{~d})$ tomato purée consumption (8.2 (SEM 2.8)\% after $24 \mathrm{~h}$ and 8.3 (sem $2 \cdot 7) \%$ after $7 \mathrm{~d}$ of intervention with tomato purée). In addition, we found no significant differences in FMD between the tomato and control periods at any time point investigated (after 24h: 8.6 (SEM 3.5)\% for control and 8.2 (SEm $2 \cdot 8) \%$ for the tomato period; after $7 \mathrm{~d}: 8 \cdot 2(\operatorname{sem} 2 \cdot 7) \%$ for control and 8.3 (SEM 2.7 ) $\%$ for the tomato period). Finally, there was no significant correlation between

Table 1. Baseline characteristics of the study population ( $n$ 19)

(Mean values and standard deviations)

\begin{tabular}{lcc}
\hline Variables & Mean & SD \\
\hline Age (years) & $58 \cdot 9$ & $6 \cdot 3$ \\
Systolic blood pressure $(\mathrm{mmHg})$ & 115 & 10 \\
Diastolic blood pressure $(\mathrm{mmHg})$ & 70 & 6 \\
BMI $\left(\mathrm{kg} / \mathrm{m}^{2}\right)$ & $25 \cdot 0$ & $3 \cdot 3$ \\
Heart rate $($ beats/min) & 61 & 7 \\
Total cholesterol $(\mathrm{mg} / \mathrm{l})$ & 1990 & 290 \\
HDL-cholesterol $(\mathrm{mg} / \mathrm{l})$ & 790 & 170 \\
LDL-cholesterol $(\mathrm{mg} / \mathrm{l})$ & 1220 & 260 \\
TAG $(\mathrm{mg} / \mathrm{l})$ & 790 & 230 \\
HbA1c $(\%)$ & $5 \cdot 6$ & $0 \cdot 3$ \\
Homocysteine $(\mu \mathrm{m})$ & $9 \cdot 4$ & $2 \cdot 1$ \\
C-reactive protein $(\mathrm{mg} / \mathrm{l})$ & $1 \cdot 3$ & $1 \cdot 3$ \\
\hline
\end{tabular}

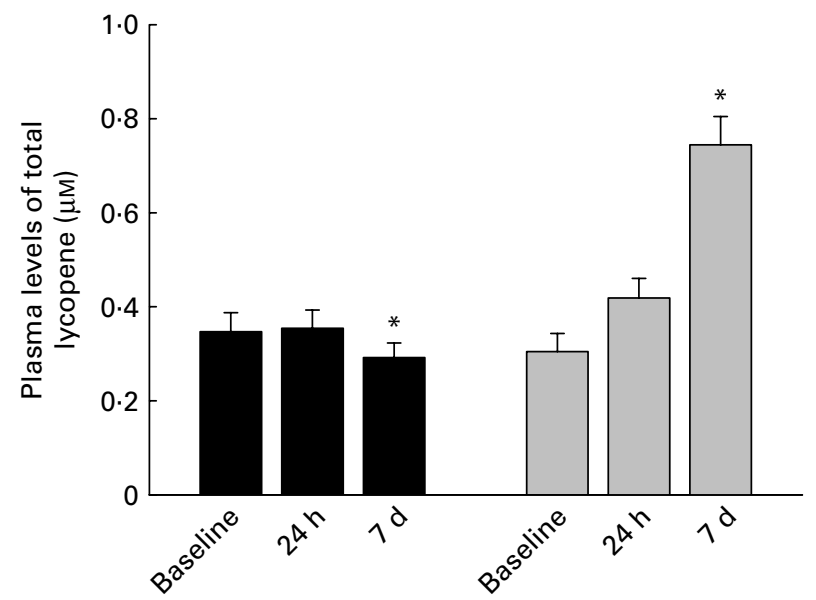

Fig. 1. Time course of lycopene plasma levels. Plasma levels of lycopene at baseline, $24 \mathrm{~h}$, and $7 \mathrm{~d}$ after ingestion of a buttered roll with $70 \mathrm{~g}$ tomato purée/d (tomato period, $\square$ ) or without tomato purée (control period, 口). Data are means with their standard errors $(n 19)$. * Values were significantly different $(P<0.05)$, as calculated by linear mixed models.

lycopene plasma levels and FMD (Fig. 2). Likewise, endothelium-independent nitro-mediated dilation was not modulated by tomato consumption (data not shown).

\section{Discussion}

In a randomised, cross-over dietary intervention study, healthy non-smoking postmenopausal women consumed lycopene from tomato purée for 1 week. Although lycopene was absorbed and resulted in a significant increase in lycopene plasma levels compared with baseline and the control period (no tomato purée), endothelial-dependent vasodilation was not influenced by this intervention.

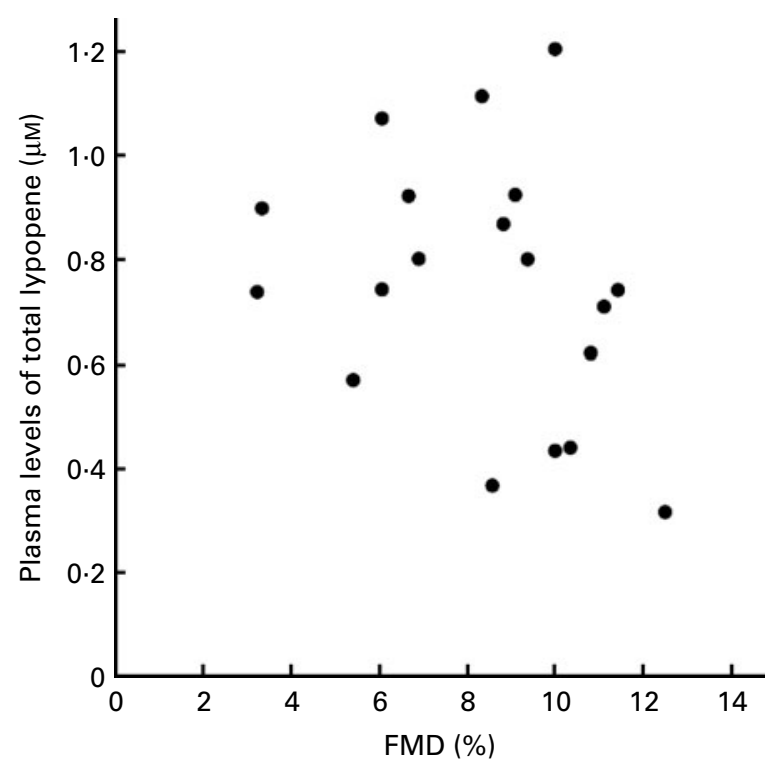

Fig. 2. Correlation between plasma lycopene levels and flow-mediated dilation at day 7 after daily consumption of $70 \mathrm{~g}$ tomato purée. There was no significant correlation between plasma lycopene levels and flow-mediated dilation (FMD). 
Experimental evidence suggests that lycopene may ameliorate the endothelial function in animal models. Suganuma \& Inakuma ${ }^{(16)}$ reported that dietary ingestion of tomato protected hypercholesterolaemic mice from endothelial dysfunction. The uptake of tomato inhibited the increase of lipid peroxides in mouse plasma in their study, suggesting that lycopene may act primarily as an antioxidant in plasma. It is well established that enhanced production or attenuated degradation of reactive oxygen species leads to oxidative stress, a process that affects endothelial function. Reactive oxygen species can interact with and inactivate NO, which is produced by the normal vascular endothelium and is a principal determinant of normal endothelial vascular function. Reduced NO bioavailability results in endothelial dysfunction and increased risk of $\mathrm{CVD}^{(23)}$. FMD assessment for endothelial function is regarded as a predictor for future cardiovascular events $^{(24)}$. Purified lycopene supplementation decreased biomarkers of oxidative stress in human subjects ${ }^{(9)}$. Very recently, an inverse relationship between lycopene plasma concentration and arterial stiffness as well as oxLDL has been shown in healthy women ${ }^{(25)}$. However, the increase in lycopene plasma levels did not affect plasma lipophilic antioxidant capacity in the present study (data not shown).

Several reasons for the missing effects of tomato purée on endothelial function in the present study may be discussed. First, it is conceivable that the present study group was 'too healthy' and basal endothelial function too good to enable detection of significant effects. Since antioxidant mechanisms are very much in the foreground of lycopene properties, situations in which increased oxidative stress and subsequent endothelial dysfunction are present - such as observed in chronic smokers - might have shown different results. In addition, the inclusion of women only could have biased the outcome.

Another limitation of the present study represents the duration of the intervention time. It may have been too short, and chronic effects after long-term consumption may play a role in the vasculoprotective effects of tomato products. In a human intervention study, patients with type 2 diabetes were supplemented with tomato juice for 4 weeks. The intervention resulted in a nearly threefold increase in plasma lycopene levels. The authors found an increase in the lag time of $\mathrm{Cu}$-induced oxidation of isolated LDL ex vivo. However, cardiovascular parameters in vivo (plasma lipid levels, blood pressure, levels of C-reactive protein and circulating pro-inflammatory cell adhesion molecules) did not change significantly after intervention in this study ${ }^{(26)}$. Although a fair number of epidemiological indications for beneficial effects of tomato products on the cardiovascular system are available, sound data on intervention studies are sparse. The rationale for the rather short intervention time in the present study was based on other studies with plant-derived interventions. Consumption of red and white wine resulted in significant improvement in endothelial function (FMD) after $3 \mathrm{~h}$ in subjects with coronary artery disease ${ }^{(27)}$. Likewise, green and black tea improved FMD in healthy postmenopausal women - the same kind of subjects as employed in the present study - already after $2 \mathrm{~h}^{(20)}$. In healthy males, ingestion of a flavanol-rich cocoa drink increased FMD $1-4 \mathrm{~h}$ after consumption ${ }^{(28)}$. Even more pronounced, an acute (after $2 \mathrm{~h}$ ) as well as a long-term, sustained increase in FMD was observed after daily consumption of a high-flavanol cocoa drink for a period of $7 \mathrm{~d}$ in healthy male smokers ${ }^{(29)}$. These data indicate the efficacy of food-derived interventions to improve the endothelial function, both after shorter intervention times and in healthy subjects.

Clearly, there is a difference in the time and mode of action between the above-mentioned foods and tomato products in terms of endothelial function measured as FMD. The potential reasons for this discrepancy can only be speculated upon. It may be attributed to the fact that hydrophilic water-soluble polyphenols may influence the cellular processes directly and in a rather short time via activation of cellular surface receptors. Lipophilic carotenoids are mainly transported within lipoproteins in the blood $^{(30)}$. It can be argued that their hydrophobic nature prevents the direct access to cell receptors and hence immediate cellular responses. Plasma kinetics also differ between polyphenols and lipophilic carotenoids. While plant-derived polyphenols are adsorbed rapidly in the blood within a few hours, carotenoids require a longer time of adsorption. Thus, much longer intervention times might be required to observe the physiological effects with tomato products. However, since we found slightly elevated plasma lycopene levels already after $24 \mathrm{~h}$, and a significant increase after $7 \mathrm{~d}$ of intervention, we feel confident that the intervention time in the present study was long enough to detect potential changes in endothelial function.

In conclusion, the present study shows that there is no vascular effect demonstrable by the measurement of FMD after intervention with tomato purée for $7 \mathrm{~d}$ in healthy postmenopausal women. Further investigation is warranted to determine whether beneficial effects may be observed in subjects exhibiting various cardiovascular risk factors known to result in endothelial dysfunction.

\section{Acknowledgements}

The authors thank Christian Gafert, Marco Russo, Aline Höfer and Vanessa Witzel for their excellent technical assistance. V. S. contributed to study conception, supervision of the study, data interpretation and writing of the manuscript. C. K. participated in the conduction of the human study and data collection. S. H. carried out the measurement of endothelial function and was involved in data generation and data analysis. N. J. participated in the experimental design, data interpretation and preparation 
of the manuscript. C. J. took part in data analysis and validation. V. B., K. F. and L. M. measured plasma lycopene levels and antioxidant capacities. In addition, they took part in writing of the manuscript. C. G. did the statistical analysis. M. L. was involved in experimental design, data interpretation, data validation and the writing of the manuscript. The authors have no conflicts of interest regarding the paper. The present work was supported by the European Community Sixth Framework Programme (IP 016213).

\section{References}

1. Street DA, Comstock GW, Salkeld RM, et al. (1994) Serum antioxidants and myocardial infarction. Are low levels of carotenoids and alpha-tocopherol risk factors for myocardial infarction? Circulation 90, 1154-1161.

2. Kohlmeier L, Kark JD, Gomez-Gracia E, et al. (1997) Lycopene and myocardial infarction risk in the EURAMIC Study. Am J Epidemiol 146, 618-626.

3. Ziedén B, Kaminskas A, Kristenson M, et al. (1999) Increased plasma 7 beta-hydroxycholesterol concentrations in a population with a high risk for cardiovascular disease. Arterioscler Thromb Vasc Biol 19, 967-971.

4. Gianetti J, Pedrinelli R, Petrucci R, et al. (2002) Inverse association between carotid intima-media thickness and the antioxidant lycopene in atherosclerosis. Am Heart $J$ 143, 467-474.

5. Levy Y, Zaltsberg H, Ben-Amotz A, et al. (2000) Dietary supplementation of a natural isomer mixture of betacarotene inhibits oxidation of LDL derived from patients with diabetes mellitus. Ann Nutr Metab 44, 54-60.

6. Upritchard JE, Sutherland WH \& Mann JI (2000) Effect of supplementation with tomato juice, vitamin E, and vitamin C on LDL oxidation and products of inflammatory activity in type 2 diabetes. Diabetes Care 23, 733-738.

7. Klebanov GI, Kapitanov AB, Teselkin Y, et al. (1998) The antioxidant properties of lycopene. Membr Cell Biol 12, 287-300.

8. Agarwal S \& Rao AV (1998) Tomato lycopene and low density lipoprotein oxidation: a human intervention study. Lipids 33, 981-984.

9. Devaraj S, Mathur S, Basu A, et al. (2008) A dose-response study on the effects of purified lycopene supplementation on biomarkers of oxidative stress. J Am Coll Nutr 27, 267-273.

10. Martin KR, Wu D \& Meydani M (2000) The effect of carotenoids on the expression of cell surface adhesion molecules and binding of monocytes to human aortic endothelial cells. Atherosclerosis 150, 265-274.

11. Hung CF, Huang TF, Chen BH, et al. (2008) Lycopene inhibits TNF-alpha-induced endothelial ICAM-1 expression and monocyte-endothelial adhesion. Eur J Pharmacol 586, $275-282$.

12. Lo HM, Hung CF, Tseng YL, et al. (2007) Lycopene binds PDGF-BB and inhibits PDGF-BB-induced intracellular signaling transduction pathway in rat smooth muscle cells. Biochem Pharmacol 74, 54-63.

13. Fuhrman B, Elis A \& Aviram M (1997) Hypocholesterolemic effect of lycopene and beta-carotene is related to suppression of cholesterol synthesis and augmentation of LDL receptor activity in macrophages. Biochem Biophys Res Commun 233, 658-662.
14. van Herpen-Broekmans WM, Klöpping-Ketelaars IA, Bots ML, et al. (2004) Serum carotenoids and vitamins in relation to markers of endothelial function and inflammation. Eur J Epidemiol 19, 915-921.

15. Hozawa A, Jacobs DR Jr, Steffes MW, et al. (2007) Relationships of circulating carotenoid concentrations with several markers of inflammation, oxidative stress, and endothelial dysfunction: the Coronary Artery Risk Development in Young Adults (CARDIA)/Young Adult Longitudinal Trends in Antioxidants (YALTA) study. Clin Chem 53, 447-455.

16. Suganuma H \& Inakuma T (1999) Protective effect of dietary tomato against endothelial dysfunction in hypercholesterolemic mice. Biosci Biotechnol Biochem 63, 78-82.

17. Bieri JG, Brown ED \& Smith JC (1985) Determination of individual carotenoids in human plasma by high performance liquid chromatography. J Liquid Chromatogr 8, 473-484.

18. Fröhlich K, Kaufmann K, Bitsch R, et al. (2006) Effects of ingestion of tomatoes, tomato juice and tomato purée on contents of lycopene isomers, tocopherols and ascorbic acid in human plasma as well as on lycopene isomer pattern. Br J Nutr 95, 734-741.

19. Craft NE, Brown ED \& Smith JC Jr (1988) Effects of storage and handling conditions on concentrations of individual carotenoids, retinal, and tocopherols in plasma. Clin Chem 34, 44-48.

20. Jochmann N, Lorenz M, Krosigk A, et al. (2008) The efficacy of black tea in ameliorating endothelial function is equivalent to that of green tea. BrJ Nutr 99, 863-868.

21. Corretti MC, Anderson TJ, Benjamin EJ, et al. (2002) Guidelines for the ultrasound assessment of endothelial-dependent flow-mediated vasodilation of the brachial artery: a report of the International Brachial Artery Reactivity Task Force. J Am Coll Cardiol 39, 257-265.

22. Pyke KE \& Tschakovsky ME (2005) The relationship between shear stress and flow-mediated dilation: implications for the assessment of endothelial function. J Physiol 568, $357-369$.

23. Cai H \& Harrison DG (2000) Endothelial dysfunction in cardiovascular diseases: the role of oxidant stress. Circ Res 87, 840-844.

24. Halcox JP, Donald AE, Ellins E, et al. (2009) Endothelial function predicts progression of carotid intima-media thickness. Circulation 119, 1005-1012.

25. Kim OY, Yoe HY, Kim HJ, et al. (2010) Independent inverse relationship between serum lycopene concentration and arterial stiffness. Atherosclerosis 208, 581-586.

26. Upritchard JE, Sutherland WH \& Mann JI (2000) Effect of supplementation with tomato juice, vitamin $\mathrm{E}$, and vitamin $\mathrm{C}$ on LDL oxidation and products of inflammatory activity in type 2 diabetes. Diabetes Care 23, 733-738.

27. Whelan AP, Sutherland WH, McCormick MP, et al. (2004) Effects of white and red wine on endothelial function in subjects with coronary artery disease. Intern Med J 34, 224-228

28. Schroeter H, Heiss C, Balzer J, et al. (2006) (-)-Epicatechin mediates beneficial effects of flavanol-rich cocoa on vascular function in humans. Proc Natl Acad Sci U S A 103, 1024-1029.

29. Heiss C, Finis D, Kleinbongard P, et al. (2007) Sustained increase in flow-mediated dilation after daily intake of high-flavanol cocoa drink over 1 week. J Cardiovasc Pharmacol 49, 74-80.

30. Goulinet S \& Chapman MJ (1997) Plasma LDL and HDL subspecies are heterogenous in particle content of tocopherols and oxygenated and hydrocarbon carotenoids. Relevance to oxidative resistance and atherogenesis. Arterioscler Thromb Vasc Biol 17, 786-796. 